\title{
New antibacterial dihydrochalcone derivatives from buds of Populus balsamifera
}

Serge Lavoie ${ }^{\mathrm{a}}$, Jean Legault ${ }^{\mathrm{a}}$, François Simard ${ }^{\mathrm{a}}$, Éric Chiasson ${ }^{\mathrm{a}}$ and André Pichette, ${ }^{\mathrm{a}, *}$

a Département des Sciences Fondamentales, Université du Québec à Chicoutimi, Chicoutimi, Québec, Canada, G7H 2B1

* Corresponding author. Tel.: +1 418545 5011; fax: +1 418545 5012. E-mail address: andre_pichette@uqac.ca (A. Pichette).

\section{Graphical abstract}

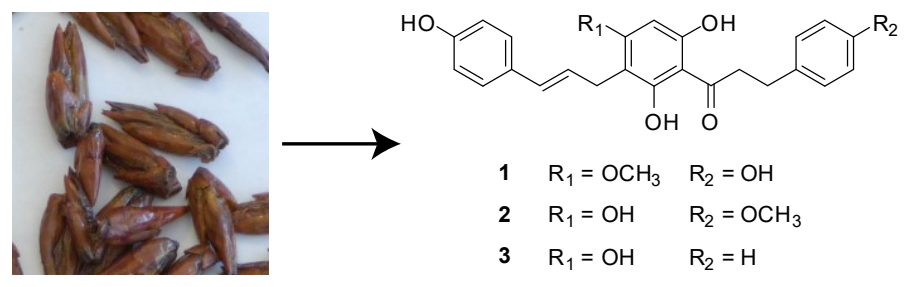




\begin{abstract}
Three new dihydrochalcone derivatives, balsacones A, B and C, along with seven known compounds, were isolated from the buds of Populus balsamifera. The structures of the new compounds were elucidated by the analysis of spectroscopic data. Only balsacone A, B and C were significantly active against Staphylococcus aureus with MIC values ranging from 3.1 to $6.3 \mu \mathrm{M}$.
\end{abstract}

\title{
Keywords
}

Dihydrochalcone, Populus balsamifera, Antibacterial activity, Staphylococcus aureus, NMR 


\section{Introduction}

Populus balsamifera L., belonging to the Salicaceae family, is a tree inhabiting almost all part of North America ${ }^{1}$. The American aboriginals prepared ointment from its buds to treat wounds ${ }^{2}$ suggesting that this substance might act as a protection against infections. This traditional use, combined to recent results indicating that buds extracts from other Populus species possess antimicrobial activity, ${ }^{3}$ prompted new investigation on a $P$. balsamifera buds extract to identify antimicrobial compounds. Previous phytochemical studies on $P$. balsamifera buds resulted in the identification of alkanes, ${ }^{4}$ fatty acids, ${ }^{4,5}$ terpenes, ${ }^{4,6}$ phenols, ${ }^{4,7,8}$ flavonoids,,${ }^{4,7-9}$ chalcones, ${ }^{4,7-9}$ carbohydrates ${ }^{10}$ and prostaglandins. ${ }^{11}$ Herein are reported the isolation and structural elucidation of three new dihydrochalcone derivatives, characterized as balsacones A-C (1-3), together with seven known compounds (Figure 1). All isolates were tested for biological activities against Staphylococcus aureus, Escherichia coli and normal skin fibloblast (WS1).

\section{Results and discussion}

Crushed and air-dried buds of $P$. balsamifera $(875 \mathrm{~g})$ were extracted with refluxing aqueous ethanol of increasing polarity $\left(5 \%\right.$ to $\left.30 \% \mathrm{H}_{2} \mathrm{O}\right)$. Solvent partition of the combined aqueous EtOH extracts followed by column chromatographic separations and semi-preparative HPLC purifications resulted in the isolation of compounds 1-10.

Compound 1 was isolated as an orange amorphous solid which gave a $[\mathrm{M}+\mathrm{K}]^{+}$ quasimolecular ion peak at $m / z 459.1228$ in the HRMS spectrum consistent with the molecular formula $\mathrm{C}_{25} \mathrm{H}_{24} \mathrm{O}_{6} .{ }^{12}$ The IR spectrum showed bands at 3354 and $1612 \mathrm{~cm}^{-1}$, due to $\mathrm{OH}$ and a conjugated carbonyl respectively. The ${ }^{13} \mathrm{C}$ and DEPT-135 NMR spectra showed the presence of 21 carbon resonances accounting for one carbonyl, five oxygenated aromatic quaternaries, seven $\mathrm{sp}^{2}$ methines, four aromatic quaternaries, one oxygenated methyl and three aliphatic methylenes (Table 1). Detail analysis of $1 \mathrm{D}{ }^{1} \mathrm{H}$, 2D-COSY and HSQC experiments (Figure 2) showed signals for two 1,4-disubstituted aromatic rings at $\delta_{\mathrm{H}} 7.05(2 \mathrm{H}, \mathrm{d}, \mathrm{J}=8.3 \mathrm{~Hz}), 6.69(2 \mathrm{H}, \mathrm{d}, \mathrm{J}=8.3 \mathrm{~Hz})$ and $7.11(2 \mathrm{H}, \mathrm{d}, \mathrm{J}=$ $8.5 \mathrm{~Hz}), 6.66(2 \mathrm{H}, \mathrm{d}, \mathrm{J}=8.5 \mathrm{~Hz})$. Two other spin systems could be observed in COSY, an allyl at $6.23(1 \mathrm{H}, \mathrm{br} \mathrm{d}, \mathrm{J}=15.6 \mathrm{~Hz}), 6.05(1 \mathrm{H}, \mathrm{dt}, \mathrm{J}=15.6,6.3 \mathrm{~Hz})$ and $3.36(2 \mathrm{H}, \mathrm{m})$ and two methylenes at $3.31(2 \mathrm{H}, \mathrm{m})$ and $2.87(2 \mathrm{H}, \mathrm{t}, \mathrm{J}=8.2 \mathrm{~Hz})$. The HMBC correlations of $\mathrm{H}-2$ with $\delta_{\mathrm{C}} 156.5$ (C-4) and 31.5 (C-7) and of H-7 and H-8 with $\delta_{\mathrm{C}} 207.1$ (C-9) suggested that the first ring was hydroxylated and branched to a chain consisting of two methylenes and a carbonyl. HMBC signals of H-2" with $\delta_{\mathrm{C}} 157.4$ (C-4") and 130.3 (C-7") showed that the second ring was also hydroxylated and branched to the allyl group. The six remaining carbons were assigned to a third aromatic ring substituted by three oxygenated functions and the two phenylpropane groups described above. The exact positions of all the substituents were determined from the HMBC correlations of H-9" with $\delta_{\mathrm{C}} 165.1$ and 164.0 and the methoxy group with $\delta_{\mathrm{C}} 165.1$. From these data, only two possibilities can be considered for the position of the methoxy group: C-2' or C-4'. Using 
1D-selective NOESY experiment, a clear correlation between the methoxy group and H5' was observed meaning that the methoxy group was branched at C-4'. Examination of the mass spectrum (APCI) revealed a fragment at $\mathrm{m} / \mathrm{z} 301$ (Figure 2) thus confirming the proposed structure. Based on the above spectral evidence, 1 was characterized as 4,2',6'trihydroxy-3'-(4"-hydroxycinnamyl)-4'-methoxydihydrochalcone and named balsacone A.

Compound 2 was isolated as an orange amorphous solid which gave a $[\mathrm{M}+\mathrm{K}]^{+}$ quasimolecular ion peak at $m / z 459.1209$ in the HRMS spectrum consistent with the molecular formula $\mathrm{C}_{25} \mathrm{H}_{24} \mathrm{O}_{6} .{ }^{13}$ The IR spectrum showed bands at 3335 and $1611 \mathrm{~cm}^{-1}$, due to $\mathrm{OH}$ and conjugated carbonyl respectively. The ${ }^{13} \mathrm{C}$ and DEPT-135 NMR spectra afforded signals accounting for 21 carbons (one carbonyl, five oxygenated armomatic quaternaries, seven $\mathrm{sp}^{2}$ methines, four aromatic quaternaries, one oxygenated methyl and three aliphatic methylenes). The ${ }^{1} \mathrm{H}$ and ${ }^{13} \mathrm{C}$ NMR spectra were similar to those of compound 1 suggesting that $\mathbf{2}$ was an isomer. Indeed, after detail analysis of NMR spectra, the only difference was accounted for the position of the methoxy group, which was determined as $\mathrm{C}-4$ by the $\mathrm{HMBC}$ correlation at $\delta_{\mathrm{H}} 3.73\left(3 \mathrm{H}, \mathrm{s}, \mathrm{OCH}_{3}\right)$ and $\delta_{\mathrm{C}} 159.4$ (C-4). Compound 2 was thus characterized as 2',4',6'-trihydroxy-3'-(4"hydroxycinnamyl)-4-methoxydihydrochalcone and named balsacone B.

Compound 3 was isolated as an orange amorphous solid which gave a $[\mathrm{M}+\mathrm{H}]^{+}$ quasimolecular ion peak at $m / z 391.1500$ in the HRMS spectrum consistent with the molecular formula $\mathrm{C}_{24} \mathrm{H}_{22} \mathrm{O}_{5} .{ }^{14}$ The IR spectrum showed bands at 3332 and $1611 \mathrm{~cm}^{-1}$, due to $\mathrm{OH}$ and conjugated carbonyl respectively. The ${ }^{13} \mathrm{C}$ and DEPT-135 NMR spectra afforded signals accounting for 20 carbons (one carbonyl, four oxygenated aromatic quaternaries, eight $\mathrm{sp}^{2}$ methines, four aromatic quaternaries and three aliphatic methylenes). Detail analysis of 1D ${ }^{1} \mathrm{H}, 2 \mathrm{D}-\mathrm{COSY}$ and HSQC experiments showed signals for one mono-substituted aromatic at $\delta_{\mathrm{H}} 7.23(2 \mathrm{H}, \mathrm{m}), 7.23(2 \mathrm{H}, \mathrm{m})$ and $7.14(1 \mathrm{H}, \mathrm{m})$ and one 1,4-disubstituted aromatic ring at $\delta_{\mathrm{H}} 7.12(2 \mathrm{H}, \mathrm{m})$ and $6.66(2 \mathrm{H}, \mathrm{d}, \mathrm{J}=8.5 \mathrm{~Hz})$. The remaining signals were almost identical to those of compound $\mathbf{1}$ and $\mathbf{2}$. Compound $\mathbf{3}$ was thus characterized as 2',4',6'-trihydroxy-3'-(4"-hydroxycinnamyl)-dihydrochalcone and named balsacone $\mathrm{C}$.

All other isolates were known substances identified as trans-cinnamic acid (4), ${ }^{15}$ trans-4-hydroxycinnamic acid (5), ${ }^{16} 4,2^{\prime}, 6^{\prime}$-trihydroxy-4'-methoxydihydrochalcone (6), ${ }^{17}$ 2',4',6'-trihydroxy-4-methoxydihydrochalcone (7), ${ }^{17}$ 2',4',6'-trihydroxydihydrochalcone (8), ${ }^{18} 2^{\prime}, 6^{\prime}$-dihydroxy-4,4'-dimethoxydihydrochalcone (9) ${ }^{19}$ and 2',6'-dihydroxy-4'methoxydihydrochalcone (10). ${ }^{20}$

Conserva and coworkers proposed a biosynthetic pathway for similar compounds isolated from Iryanthera laevis in $1990 .{ }^{21}$ From their results, the biosynthesis of the balsacones A, B and C could be explained by cinnamylation reactions of compounds 6,7 and $\mathbf{8}$ respectively. Indeed, Conserva et al. supposed the existence of compound $\mathbf{2}$ without being able to isolate it. 
Antibacterial activity of compounds 1-10 were evaluated against E. coli (gram-) and $S$. aureus $\left(\mathrm{gram}^{+}\right)$. The results presented in Table 2 show that all compounds were inactive against $E$. coli. In contrast, dihydrochalcones 1-3 were found significantly active against $S$. aureus with MIC values of $6.3 \mu \mathrm{M}, 6.3 \mu \mathrm{M}$ and $3.1 \mu \mathrm{M}$, respectively. All other compounds (4-10) were inactive with MIC higher than $100 \mu \mathrm{M}$. These results suggest that the presence of the 4-hydroxycinnamyl group at position 3' of ring A of balsacones (1-3) is important to obtain significant biological activities. Indeed, compounds 6-10, which are missing the 4-hydroxycinnamyl group, are inactive. Hufford and Oguntimein reported also that the presence of an alkyl group in position $3^{\prime}$ of dihydrochalcone ring $\mathrm{A}$ is essential for antibacterial activity. ${ }^{22}$ In addition, Awouafack and coworkers suggested that a prenyl group at the same position is an antibacterial inducer. ${ }^{23}$ Surprisingly, their compounds were active against $E$. coli, but not against $S$. Aureus suggesting that the structure of alkyl groups could modulate the selectivity toward gram + or gram- bacteria. The cytotoxicity of compounds 1-10 was also evaluated on human skin fibroblasts, WS1. The results presented in Table 2 show that compounds $\mathbf{1 , 3 , 8 - 1 0}$ were moderately cytotoxic with $\mathrm{IC}_{50}$ values ranging from 20 to $35 \mu \mathrm{M}$. Interestingly, compound $\mathbf{2}$ possesses antibacterial properties without cytotoxicity on WS1 cells.

\section{Acknowledgments}

This study was supported by a FQRNT fonds forestier 02 grant. The authors also wish to thank Marcel Pichette for buds collection, Guillaume Desbiens for extraction and Catherine Dussault for biological evaluation.

\section{References and notes}

1. Marie-Victorin, F.E.C. La flore Laurentienne, 3rd ed.; Les presses de l'Université de Montréal: Montréal, Québec, 1995; pp 162.

2. Van Wyk, B.-E.; Wink, M. Medicinal plants of the world; Timber Press, 2004.

3. Vardar-Ünlü, G.; Silici, S.; Ünlü, M. World J. Microbiol. Biotechnol. 2008, 24(7), 1011-1017.

4. Isidorov, V.; Vinogorova, V. T. Z. Naturforsch C: biosci. 2003, 58, 355-360.

5. Polyakov, V. V.; Orlov, V. K.; Shukenova, R. Z.; Mullaeva, N. I. Chem. Nat. Compd. 1985, 21, 795.

6. Mattes, B. R.; Clausen, T. P.; Reichardt, P. B. Phytochemistry 1987, 26, 13611366.

7. Sentsov, M. F.; Braslovskii, V. B.; Kurkin, V. A.; Zapesochnaya, G. G.; Bakulin, V. T.; Pravdivtseva, O. E. Rastitel'nye Resursy 1997, 33, 51-56.

8. Greenaway, W.; May, J.; Whatley, F. R. J. Chromatogr. 1989, 472, 393-400.

9. Kurkin, V. A.; Zapesochnaya, G. G.; Braslavskii, V. B. Chem. Nat. Compd. 1990, 26, 224-225.

10. Isaeva, E. V.; Ryazanova, T. V. Khimiya Rastitel'nogo Syr'ya 2006, 33-36. 
11. Levin, E. D.; Isaeva, E. V.; Cherepanova, V. E. Phytochemistry 1990, 29, 23252326.

12. Balsacone A (1): orange amorphous solid; UV (MeOH) $\lambda_{\max }$ 194, 208 (sh), 268, $288 \mathrm{~nm}$; IR (film) $v_{\max } 3354,2925,1612,1513,1446,1420,1220,1138,1106$, 968, $830 \mathrm{~cm}^{-1}$; ${ }^{1} \mathrm{H}$ NMR and ${ }^{13} \mathrm{C}$ NMR, see Table 1; APCIMS (pos.) $\mathrm{m} / \mathrm{z} 421$ [M$\mathrm{H}]^{+}$(21), 301 (100); HRMS $m / z 459.12276$ (calcd for $\mathrm{C}_{25} \mathrm{H}_{24} \mathrm{O}_{6} \mathrm{~K}$, 459.12099).

13. Balsacone B (2): orange amorphous solid; UV (MeOH) $\lambda_{\max }$ 194, 208 (sh), 268, $288 \mathrm{~nm}$; IR (film) $v_{\max } 3335,2930,1611,1512,1438,1244,1176,1137,828 \mathrm{~cm}^{-1}$; ${ }^{1} \mathrm{H}$ NMR and ${ }^{13} \mathrm{C}$ NMR, see Table 1; APCIMS (pos.) $m / z 421[\mathrm{M}-\mathrm{H}]^{+}$(43), 301 (100); HRMS $m / z 459.12085$ (calcd for $\mathrm{C}_{25} \mathrm{H}_{24} \mathrm{O}_{6} \mathrm{~K}, 459.12099$ ).

14. Balsacone C (3): orange amorphous solid; UV (MeOH) $\lambda_{\max }$ 192, 206 (sh), 266, $290 \mathrm{~nm}$; IR (film) $v_{\max } 3332,2919,1611,1512,1442,1250,1217,1139,1080$, $833 \mathrm{~cm}^{-1}$; ${ }^{1} \mathrm{H}$ NMR and ${ }^{13} \mathrm{C}$ NMR, see Table 1; APCIMS (pos.) $\mathrm{m} / z 391[\mathrm{M}-\mathrm{H}]^{+}$ (36), 271 (100). HRMS $m / z 391.15000$ (calcd for $\mathrm{C}_{24} \mathrm{H}_{23} \mathrm{O}_{5}, 391.15455$ ).

15. Kalinowska, M.; Swislocka, R.; Lewandowski, W. J. Mol. Struct. 2007, 834-836, 572-580.

16. Massow, F.; Smith, M. A. R. J. Chem. Soc., Perkin Trans. 2 1976, 977-980.

17. Mizuno, M.; Kojima, H.; Tanaka, T.; Iinuma, M.; Kimura, R.; Zhi-Da, M.; Murata, H. Phytochemistry 1987, 26, 2071-2074.

18. Mustafa, K.; Kjaergaard, H. G.; Perry, N. B.; Weavers, R. T. Tetrahedron 2003, 59, 6113-6120.

19. Nilsson, M. Acta Chem. Scand. 1961, 15, 154-158.

20. Tanaka, H.; Ichino, K.; Ito, K. Phytochemistry 1984, 23, 1198-1199.

21. Conserva, L. M.; Yoshida, M.; Gottlieb, O. R.; Martinez V., J. C.; Gottlieb, H. E. Phytochemistry 1990, 29, 3911-3918.

22. Hufford, C.D.; Oguntimein, B.O. J. Nat. Prod. 1982, 45(3), 337-342.

23. Awouafack, M.D.; Kusari, S.; Lamshöft, M.; Ngamga, D.; Tane, P.; Spiteller, M. Planta med. 2010, 76(6), 640-643.

\section{Supplementary material}

Supplementary data (Experimental details on the isolation of 1-3 and their spectral data) associated with this article can be found, in the online version, at doi: 


\section{Table 1}

${ }^{1} \mathrm{H}(400 \mathrm{MHz})$ and ${ }^{13} \mathrm{C}(100 \mathrm{MHz})$ NMR data for balsacones $\mathrm{A}(\mathbf{1}), \mathrm{B}(\mathbf{2})$ and $\mathrm{C}(\mathbf{3})$ in $\mathrm{CDCl}_{3}$

\begin{tabular}{|c|c|c|c|c|c|c|}
\hline \multirow[b]{2}{*}{ no. } & \multicolumn{2}{|r|}{1} & \multicolumn{2}{|r|}{2} & \multicolumn{2}{|r|}{3} \\
\hline & $\delta_{\mathrm{C}}^{\mathrm{a}}$ & $\delta_{\mathrm{H}}^{\mathrm{a}, \mathrm{b}}$ & $\delta_{\mathrm{C}}^{\mathrm{a}}$ & $\delta_{\mathrm{H}}^{\mathrm{a}, \mathrm{b}}$ & $\delta_{\mathrm{C}}^{\mathrm{a}}$ & $\delta_{\mathrm{H}}^{\mathrm{a}, \mathrm{b}}$ \\
\hline 1 & $134.0(\mathrm{~s})$ & & $135.2(\mathrm{~s})$ & & $143.3(\mathrm{~s})$ & \\
\hline 2,6 & $130.4(d)$ & $7.05(2 \mathrm{H}, \mathrm{d}, 8.4)$ & $130.4(d)$ & $7.13(2 \mathrm{H}, \mathrm{d}, 8.4)$ & 129.5 (d) & $7.23(2 \mathrm{H}, \mathrm{m})$ \\
\hline 3,5 & 116.2 (d) & $6.69(2 \mathrm{H}, \mathrm{d}, 8.4)$ & 114.8 (d) & $6.80(2 \mathrm{H}, \mathrm{d}, 8.4)$ & 129.4 (d) & $7.23(2 \mathrm{H}, \mathrm{m})$ \\
\hline 4 & $156.5(\mathrm{~s})$ & & $159.4(\mathrm{~s})$ & & 126.9 (d) & $7.14(1 \mathrm{H}, \mathrm{m})$ \\
\hline 7 & $31.5(\mathrm{t})$ & $2.86(2 \mathrm{H}, \mathrm{m})$ & $31.6(t)$ & $2.88(2 \mathrm{H}, \mathrm{m})$ & $32.4(\mathrm{t})$ & $2.95(2 \mathrm{H}, \mathrm{t}, 8.3)$ \\
\hline 8 & $47.7(t)$ & $3.31(2 \mathrm{H}, \mathrm{m})$ & $47.4(t)$ & $3.29(2 \mathrm{H}, \mathrm{m})$ & $47.1(\mathrm{t})$ & $3.33(2 \mathrm{H}, \mathrm{m})$ \\
\hline 9 & $207.1(\mathrm{~s})$ & & $206.4(\mathrm{~s})$ & & $206.2(\mathrm{~s})$ & \\
\hline $1^{\prime}$ & $105.8(\mathrm{~s})$ & & $105.3(\mathrm{~s})$ & & $105.3(\mathrm{~s})$ & \\
\hline $2^{\prime}$ & $164.0(\mathrm{~s})$ & & $165.1(\mathrm{~s})$ & & $165.2(\mathrm{~s})$ & \\
\hline $3^{\prime}$ & $107.7(\mathrm{~s})$ & & $106.7(\mathrm{~s})$ & & $106.7(\mathrm{~s})$ & \\
\hline $4^{\prime}$ & $165.1(\mathrm{~s})$ & & $163.9(\mathrm{~s})$ & & $163.9(\mathrm{~s})$ & \\
\hline $5^{\prime}$ & $91.2(\mathrm{~d})$ & $6.04(1 \mathrm{H}, \mathrm{s})$ & $95.0(\mathrm{~d})$ & $5.94(1 \mathrm{H}, \mathrm{br} \mathrm{s})$ & $95.0(\mathrm{~d})$ & $5.94(1 \mathrm{H}$, br s $)$ \\
\hline $6^{\prime}$ & $162.4(\mathrm{~s})$ & & $161.8(\mathrm{~s})$ & & $161.8(\mathrm{~s})$ & \\
\hline $1 "$ & $131.2(\mathrm{~s})$ & & $131.3(\mathrm{~s})$ & & $131.4(\mathrm{~s})$ & \\
\hline 2",6" & $128.1(d)$ & $7.11(2 \mathrm{H}, \mathrm{d}, 8.5)$ & $128.1(\mathrm{~d})$ & $7.12(2 \mathrm{H}, \mathrm{d}, 8.4)$ & $128.1(\mathrm{~d})$ & $7.12(2 \mathrm{H}, \mathrm{m})$ \\
\hline 3",5" & $116.2(d)$ & $6.66(2 \mathrm{H}, \mathrm{d}, 8.5)$ & $116.2(d)$ & $6.66(2 \mathrm{H}, \mathrm{d}, 8.4)$ & 116.2 (d) & $6.66(2 \mathrm{H}, \mathrm{d}, 8.5)$ \\
\hline $4 "$ & $157.4(\mathrm{~s})$ & & $157.4(\mathrm{~s})$ & & $157.4(\mathrm{~s})$ & \\
\hline $7 "$ & $130.3(d)$ & $6.22(1 \mathrm{H}$, br d, 15.8) & $130.2(d)$ & $6.26(1 \mathrm{H}, \mathrm{br} \mathrm{d}, 15.7)$ & $130.2(d)$ & $6.25(1 \mathrm{H}$, br d, 15.7) \\
\hline $8 "$ & $126.6(d)$ & $6.05(1 \mathrm{H}, \mathrm{dt}, 15.8,6.3)$ & $126.8(\mathrm{~d})$ & $6.10(1 \mathrm{H}, \mathrm{dt}, 15.7,6.4)$ & $126.8(\mathrm{~d})$ & $6.11(1 \mathrm{H}, \mathrm{dt}, 15.7,6.3)$ \\
\hline $9 "$ & $26.4(t)$ & $3.35(2 \mathrm{H}, \mathrm{m})$ & $26.5(t)$ & $3.36(2 \mathrm{H}, \mathrm{m})$ & $26.5(t)$ & $3.36(2 \mathrm{H}, \mathrm{m})$ \\
\hline $\mathrm{OCH}_{3}$ & $56.1(\mathrm{q})$ & $3.83(3 \mathrm{H}, \mathrm{s})$ & $55.7(q)$ & $3.73(3 \mathrm{H}, \mathrm{s})$ & & \\
\hline
\end{tabular}


Table 2

Antibacterial and cytotoxic activities of compounds 1-10

\begin{tabular}{cccc}
\hline \multirow{2}{*}{ Compounds } & \multicolumn{2}{c}{$\mathrm{MIC}(\mu \mathrm{M})^{\mathrm{a}}$} & $\mathrm{IC}_{50}(\mu \mathrm{M})$ \\
\cline { 2 - 4 } & E. coli & S. aureus & WS1 \\
\hline $\mathbf{1}$ & $>200$ & 6.3 & $25 \pm 2$ \\
$\mathbf{2}$ & $>200$ & 6.3 & $>200$ \\
$\mathbf{3}$ & $>200$ & 3.1 & $23.6 \pm 0.8$ \\
$\mathbf{4}$ & $>200$ & $>200$ & $>200$ \\
$\mathbf{5}$ & $>200$ & $>200$ & $>200$ \\
$\mathbf{6}$ & $>200$ & 100 & $130 \pm 7$ \\
$\mathbf{7}$ & $\mathrm{NT}^{\mathrm{b}}$ & $\mathrm{NT}^{\mathrm{b}}$ & $>200$ \\
$\mathbf{8}$ & $>200$ & 100 & $35.0 \pm 0.3$ \\
$\mathbf{9}$ & $>200$ & $>200$ & $23 \pm 1$ \\
$\mathbf{1 0}$ & $>200$ & $>200$ & $20 \pm 1$ \\
Gentamicin $^{\mathrm{c}}$ & 0.04 & 0.02 & $\mathrm{NT}^{\mathrm{b}}$ \\
\hline
\end{tabular}

${ }^{a}$ Minimum inhibitory concentration. ${ }^{b}$ Not tested.'Positive control. 
Figure 1. Structure of compounds 1-10.

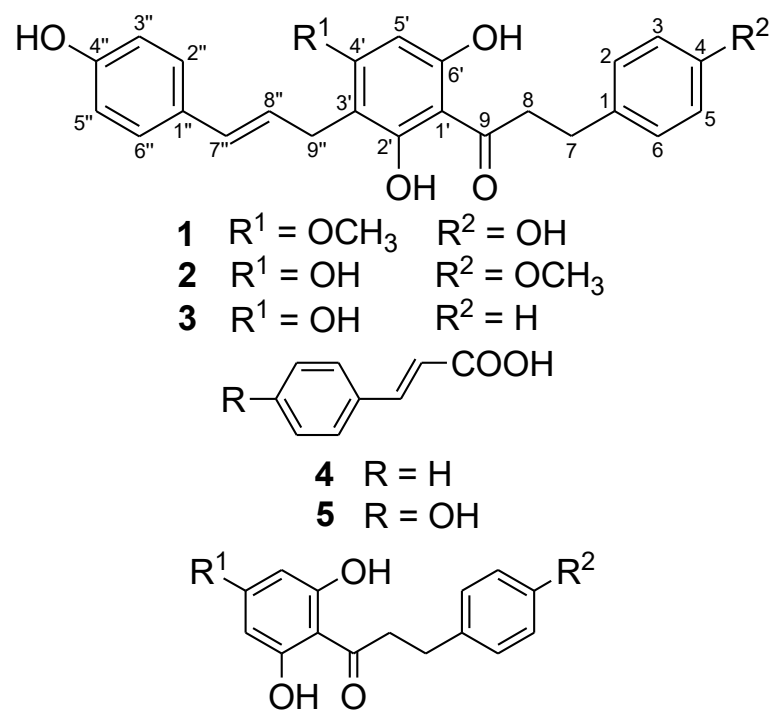

$6 \mathrm{R}^{1}=\mathrm{OCH}_{3} \quad \mathrm{R}^{2}=\mathrm{OH}$

$7 \mathrm{R}^{1}=\mathrm{OH} \quad \mathrm{R}^{2}=\mathrm{OCH}_{3}$

$8 \mathrm{R}^{1}=\mathrm{OH} \quad \mathrm{R}^{2}=\mathrm{H}$

$9 \mathrm{R}^{1}=\mathrm{OCH}_{3} \quad \mathrm{R}^{2}=\mathrm{OCH}_{3}$

$10 \mathrm{R}^{1}=\mathrm{OCH}_{3} \quad \mathrm{R}^{2}=\mathrm{H}$ 
Figure 2. COSY, HMBC, NOESY and MS key data for identification of compound 1.

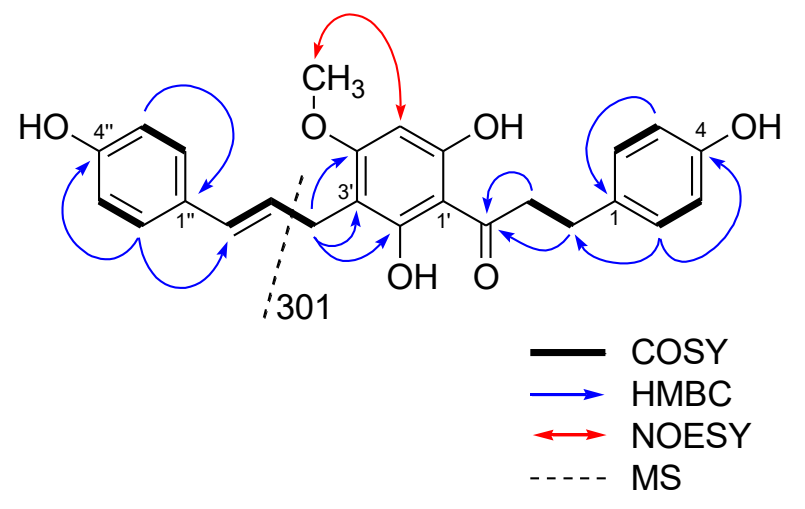

